\title{
Aspergillosis Involving the Maxillary Sinus-A Report of Two Cases and A Brief literature Review
}

\author{
Tarun Taneja, ${ }^{1}$ Susmita Saxena, ${ }^{1}$ Anita Pandey, ${ }^{2}$ Vishal Bansal, ${ }^{3}$ and Pooja Aggarwal ${ }^{1}$ \\ Departments of ${ }^{1}$ Oral Pathology and Microbiology, and ${ }^{3}$ Oral and Maxillofacial Surgery, Subharti Dental College \& \\ Hospital, Meerut, India \\ ${ }^{2}$ Department of Microbiology, Subharti Medical College \& Hospital, Meerut, India
}

Correspondence to :

Tarun Taneja

E-mail : drntaneja@ rediffmail.com

Keywords :

aspergillosis, maxillary sinus, fungal infection

\begin{abstract}
Aspergillosis is a fungal infection characterized by invasive and noninvasive forms. Noninvasive aspergillosis usually affects a healthy host, appearing either as an allergy or a cluster of fungal hyphae. Localized invasive infection of damaged tissue may be seen in a healthy host, but more extensive infection is often evident in immunocompromised patients. Its incidence in recent years has shown a marked increase and it is believed to account for many cases of nonspecific sinusitis. The purpose of this paper is to report two cases of aspergillosis involving the maxillary sinus in immunocompetent patients with an emphasis on early diagnosis because delay in the initiation of treatment can be life-threatening due to the propensity of fungi to invade adjacent blood vessels and embolize to distant organs.
\end{abstract}

\section{Introduction}

Mycotic sinusitis is on the increase worldwide. It is unclear whether this increase is due to a heightened awareness of the disease or rather to a higher incidence precipitated by the increased use of antibiotics and corticosteroids. Aspergillosis is a large spectrum of diseases caused by members of the genus Aspergillus. Although invasive fungal sinusitis may be seen in apparently healthy individuals (1, 2), Aspergillus fumigatus is most often encountered in among immunocompromised patients (3).

Aspergillus is a saprophytic mold of the Ascomycetes class that is widely distributed in the environment and is particularly found in soil, cereals, and decaying vegetation. Resistant spores are released into the air and when inhaled, may result in opportunistic fungal infection, second in frequency only to candidiasis. The individual species $A$. fumigatus, A.flavus, A. glaucis, A.terreus, and $A$. niger have been implicated in human infections, with A. fumigatus being the most common causative agent (4).

Aspergillosis appears in three forms: 1) sapro- phytic, in which there is fungal growth without invasion of viable tissue; 2) allergic, characterized by the presence of a hypersensitivity reaction to fungal hyphae or conidia ; and 3) invasive, in which there is extension of the fungus into viable tissue, resulting in severe necrosis (5). The saprophytic and allergic forms affect the immunocompetent host and have relatively low morbidity and mortality (6). Invasive or fulminant aspergillosis of the paranasal sinuses is rapidly progressive and primarily affects patients who are immunocompromised, such as those with diabetes mellitus or a malignant disease or those undergoing steroid and immunosuppressive therapy $(7,8)$.

Histopathological evaluation plays a very important role in the diagnosis. Hence this article reports two cases of aspergillosis involving the maxillary sinus in immunocompetent patients with an emphasis on early diagnosis because delay in the initiation of treatment can be life-threatening due to the propensity of fungi to invade adjacent blood vessels and embolize to distant organs. 


\section{Case Report 1}

A 24-year-old woman reported to the Department of Oral and Maxillofacial Surgery with nasal pus discharge beginning 5 months earlier. She reported that tooth \# 26 had been extracted 5 months earlier. Her medical history revealed nothing significant and the patient was found to be immunocompetent.

Intraoral examination revealed pus discharge from the socket of the extracted tooth (Fig. 1). On surgical exploration, the anterior wall of the maxillary sinus had been destroyed and the sinus was full of granulation tissue and necrotic material, diagnosed as an oro -antral fistula. The patient reported a history of debridement several times to repair the fistula.

The patient was treated by debridement and irrigation of the maxillary sinus through the oro-antral opening, following by antral packing with BIPP (bismuth iodoform paraffin packing). After 5 days, the oro-antral fistula was repaired with a buccal flap and by the Caldwell Luc approach with nasal antrostomy of the inferior meatus. During antral lavage and debridement, black granulation tissue was completely debrided and sent for histopathological and microbiological culture examination. The patient was reexamined 2 weeks later and the repeat culture was found to be negative for growth of any fungal pathogens.

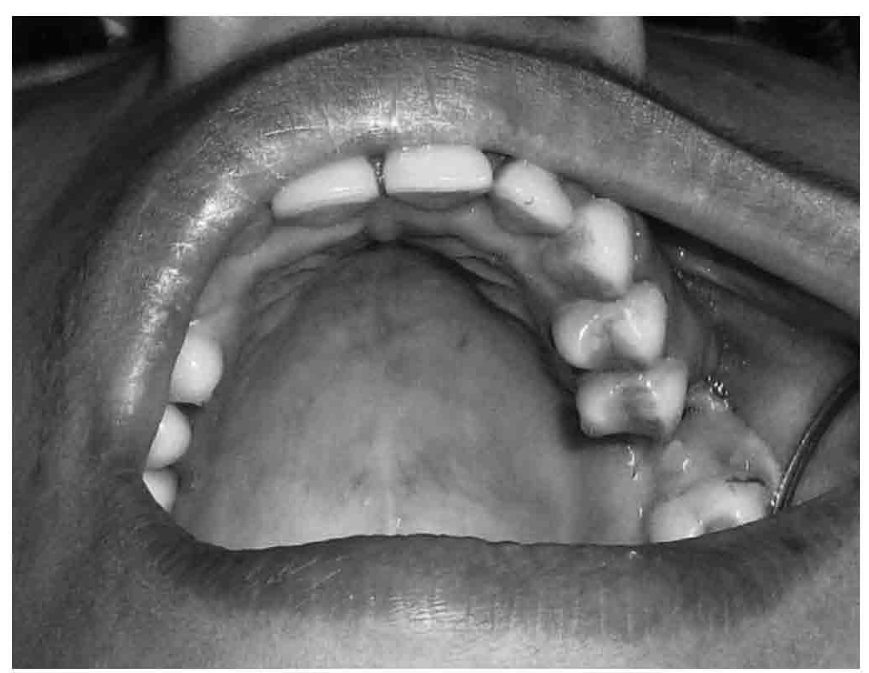

Fig. 1. Photograph of a patient (Case 1) with an oro-antral fistula with pus discharge.
The specimen received in the department of Oral Pathology and Microbiology for histopathological examination (in formalin) consisted of small black bits of soft tissue of irregular shapes and sizes. One soft tissue specimen was also received in normal saline for culture.

Histopathological examination of the specimen showed granulation tissue with intense chronic inflammation and multiple congested blood vessels. Some of the blood vessels showed eosinophilic material within the lumina. Areas of hemorrhage were evident with masses of eosinophilic, entangled hyphae-like structures and spores. Hematoxylin and eosin stain showed hyphae-like structures that were flat, broad, refractile, and dichotomously branched at a $45^{\circ}$ angle with conidiospores (Fig. 2). Necrotic areas and degenerating cells were also evident (Fig. 3). The $20 \% \mathrm{KOH}$ mount made from the tissue revealed multiple fungal hyphae.

These histopathological features were suggestive of fungal infection with the morphology of the hyphae similar to that of aspergillosis with tissue necrosis. A. fumigatus was also grown in culture on Sabouraud's dextrose agar (SDA) medium.

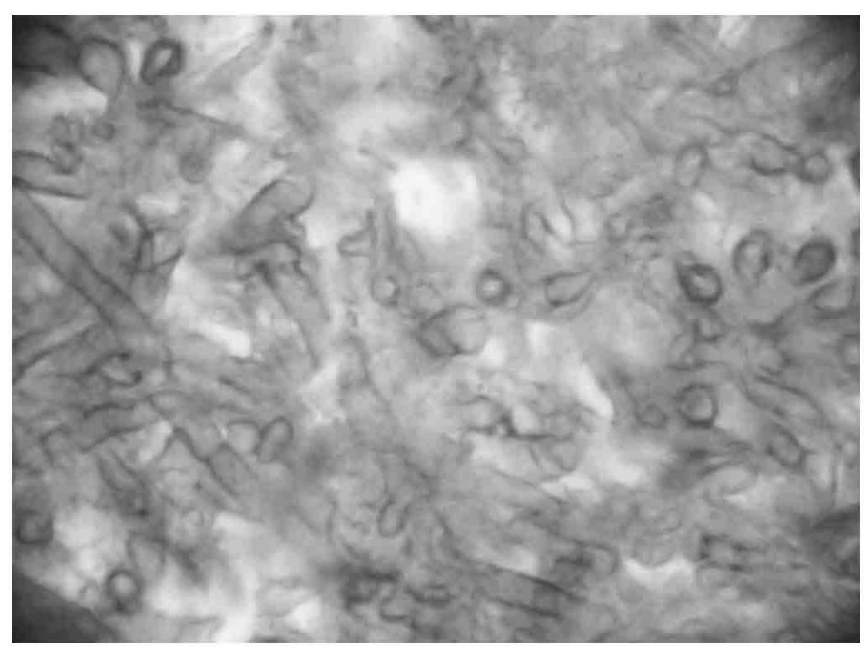

Fig. 2. Hematoxylin \& eosin stained section $(10 \times)$ showing fungal hyphae dichotomously branched at $45^{\circ}$ angles with conidiospores (Case 1). 


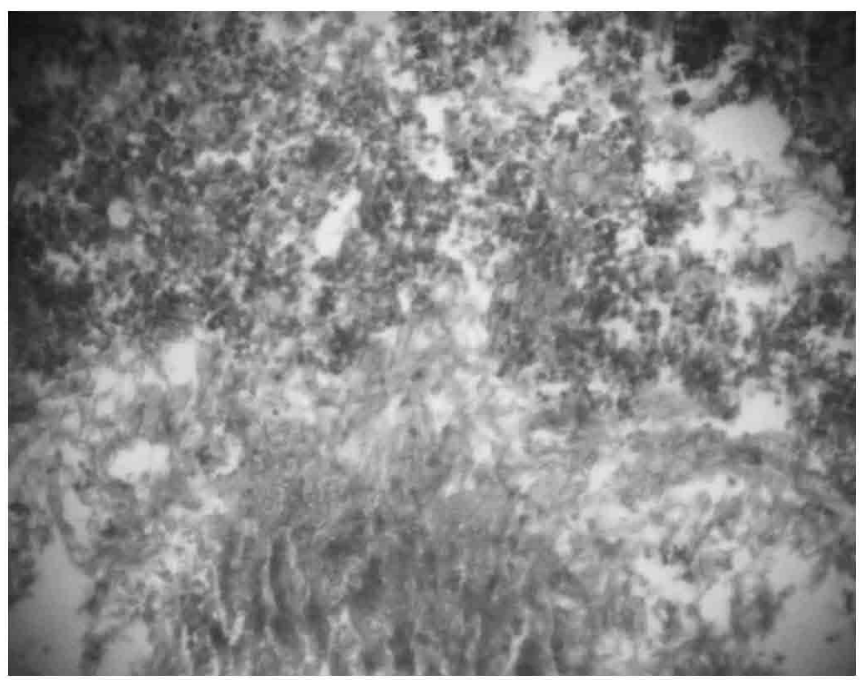

Fig. 3. Hematoxylin \& eosin stained section $(10 \times)$ showing necrosis (Case 1 )

\section{Case Report 2}

A 25-year-old woman reported to the Department of Oral and Maxillofacial Surgery with a lacerated wound in the middle third of her face extending from the left ala of the nose, involving the corner of the mouth, to the zygomatic process on the right side of her face (Fig. 4). On medical examination, the patient was found to be immunocompetent.

Under aseptic conditions, irrigation and debridement of the lacerated wound was performed, followed by suturing of the wound in layers. One month

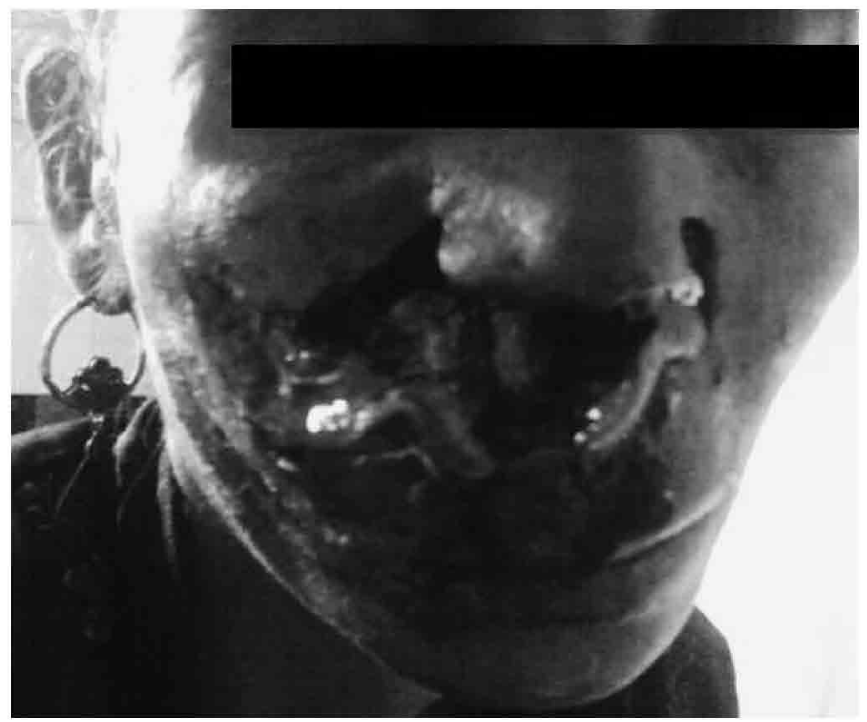

Fig. 4. Photograph of a patient (Case 2) showing a lacerated wound. later, the patient came back with pus discharge from the nose that was yellow and purulent. The patient was treated by the Caldwell Luc approach with nasal antrostomy of the inferior meatus, debridement and irrigation of the maxillary sinus through the oroantral opening, and antral packing with BIPP. During antral lavage and debridement, black granulation tissue was completely debrided and sent for histopathological and microbiological culture examination. At the follow-up examination, the patient's clinical outcome was found to be satisfactory and her culture was negative for any fungal growth.

The specimen received in the department of Oral Pathology and Microbiology for histopathological examination consisted of small multiple black bits of soft tissue of irregular shapes and sizes. Soft tissue specimen was also received in normal saline for culture.

The $20 \% \mathrm{KOH}$ mount from the tissues revealed hyphae that were septate and dichotomously branched with conidia (Fig. 5).

Hematoxylin and eosin staining of specimen sections showed an ill-defined antral lining, edematous connective tissue with mixed inflammatory cell infiltrate, and tangled masses of branched fungal hyphae with spore formation (Fig. 6). The demonstration of septate, dichotomously branched hyphae was critical in supporting the diagnosis of aspergillosis. A periodic acid-Schiff-stained section $(10 \times)$ showed tissue necrosis (Fig. 7).

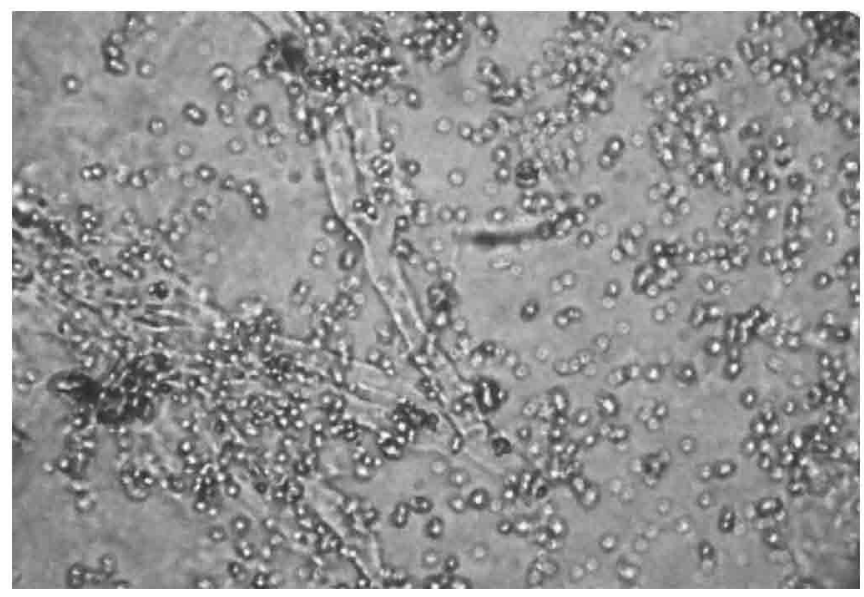

Fig. 5. Direct microscopic observation in 10\% $\mathrm{KOH}$, showing relatively small hyphae-like structures with numerous conidia (Case 2). 


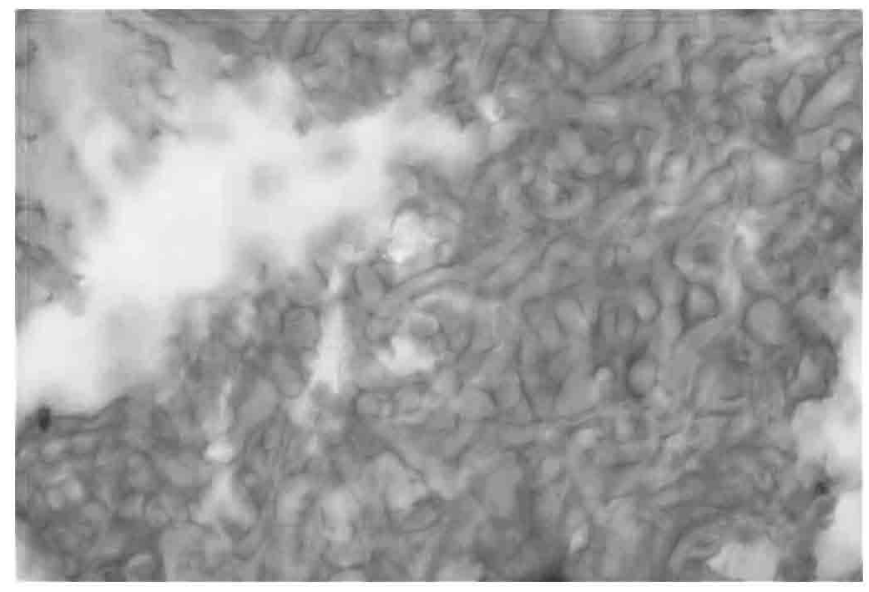

Fig. 6. Hematoxylin \& eosin stained section $(10 \times)$ showing broad refractile fungal hyphae-like structures (Case 2).

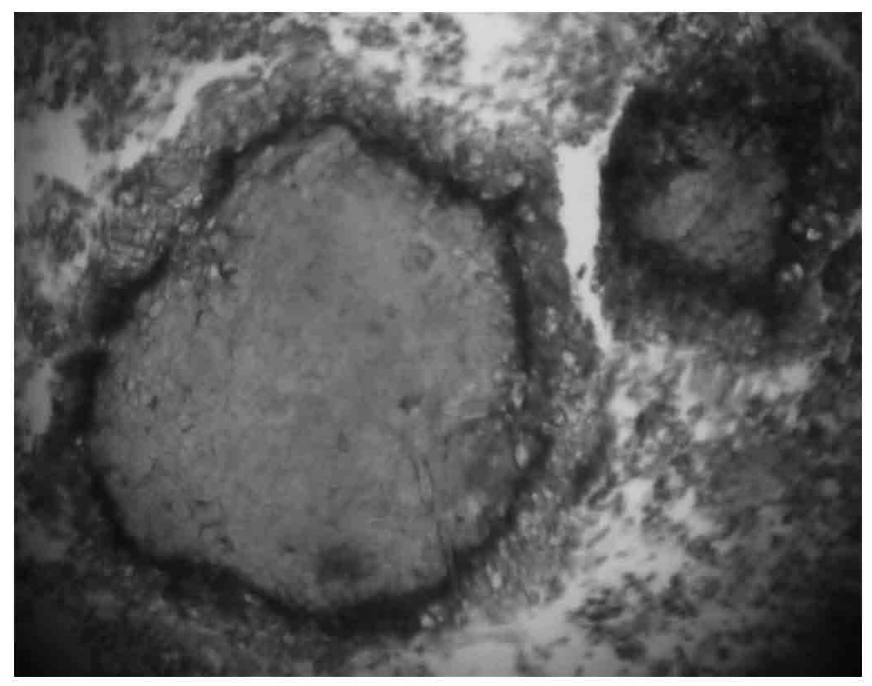

Fig. 7. Periodic acid-Schiff stained section $(10 \times)$ showing tissue necrosis (Case 2).

A. fumigatus was also grown in culture on SDA medium (Fig. 8).

\section{Discussion}

Allergic aspergillosis sinusitis was first described by Katzenstein et al. in 1984 (9) ; they likened it histologically to allergic broncho-pulmonary aspergillosis. It usually presents in young adults with a history of asthma and intranasal polyps. Its slow development is associated with symptoms that are most often nonspecific.

Aspergillosis infection of the paranasal sinuses can be noninvasive or invasive. Noninvasive aspergillosis manifests as allergic sinusitis or mycetoma and

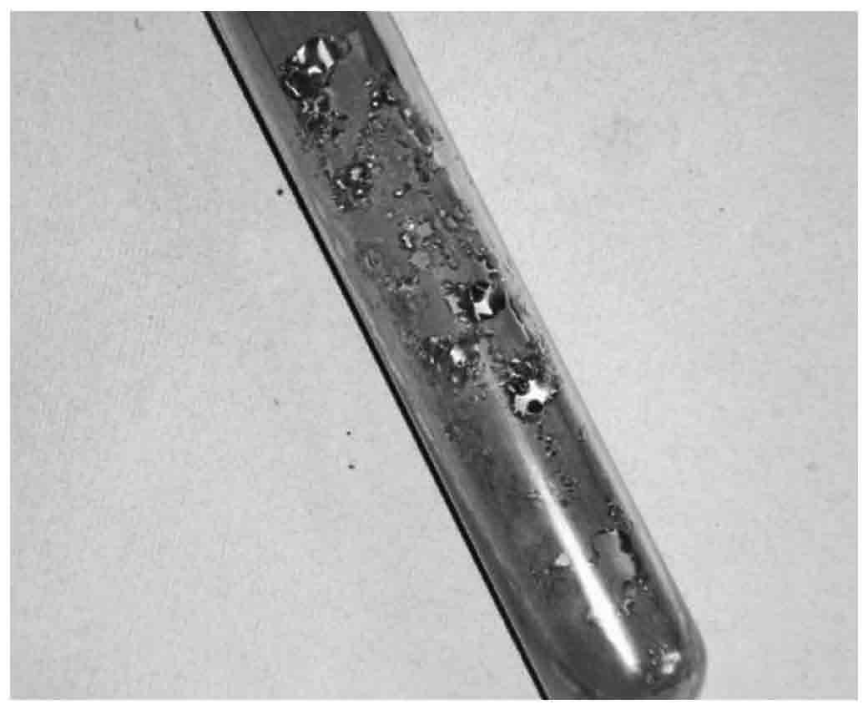

Fig. 8. Culture growth in Sabouraud's dextrose agar medium showing smoky grey-green colonies of Aspergillus fumigatus (Case 2).

is usually seen in individuals who are otherwise healthy (10), whereas invasive infection may invade into the orbit or brain and can affect multiple organs through hematogenous spread, typically encountered in immunocompromised patients $(8,11-13)$. Both of our patients had no history of diabetes and were not immunocompromised.

Although invasive sinus aspergillosis have been reported in patients who were not immunocompromised (7, 14-16), Kim et al. reported that of 36 patients with intracranial aspergillosis without immunocompromising diseases, only five survived (17). Aspergillosis involving the paranasal sinuses most often appears in the maxillary sinus $(18,19)$.

Some authors reported that tobacco might play a major role in the development of infection when no other predisposing factors existed, because it is a source of fungal spores $(16,20)$. However, neither of our patients had a history of tobacco chewing or smoking. In a healthy host, the disease often presents as an allergy or a low-grade sinus infection, resulting in the formation of a fungal mass or aspergilloma $(21,22)$. Matiaz et al. in 2004 reported a case of sinus maxillaris mycetoma of odontogenic origin caused by the leakage of endodontic material into the periapical tissues, leading to fungal growth i.e., $A$. fumigates $(2,3)$. The cause of fungal infection is 
probably poor wound in both of our cases.

The radiologic presentation of aspergillosis of the paranasal sinus is focal radiodense shadows that are usually accompanied by mucosal thickening or diffuse clouding in the sinus shown on plain radiography and computed tomography scanning. The radiographic findings are nonspecific and inconclusive and should thus be supported by lesional biopsy and cultures.

A direct microscopic examination is a rapid and cost-effective method of diagnosis, without any skewing by laboratory procedures. Some spore types are able to thrive in a particular ecological niche but may not germinate at all on laboratory media. Fast -growing spore types or those with other competitive advantages may outgrow slow-growing types or may prevent the germination of others. Some spore reservoirs may be old and may have lost viability. These spores will not germinate and will be completely missed, even though dead spores are just as antigenic as live spores for allergic persons. There are times when cultures are helpful and will be necessary, such as when speciation is desired for genera such as Aspergillus or Penicillium. Cultures of swabs or tissues should always be accompanied by a direct microscopic examination to identify the fungal species. Microscopically, Aspergillus hyphae are septate, branched, and more irregular than mucor hyphae, which are wider, pauciseptate, and flat, with branching at right angles, whereas candida hyphae are thinner, nonseptate, and are associated with yeast forms. When stained with periodic acidSchiff's stain or Gomori methanamine silver, Aspergillus hyphae measure 2 to 5 microns in width with even diameters that are frequently septate and dichotomously branched at a $45^{\circ}$ angle. Aspergilli produce conidiospores that enter the atmosphere and are inhaled into nasal and oral cavities, lungs, and paranasal sinuses.

Management of aspergillosis of the maxillary sinus involving complete debridement and curettage and the Caldwell-Luc approach with or without an intranasal antrostomy is the procedure of choice, although Stammberger et al. in 1984 recommended the use of surgery in association with local antimycotic agents such as itroconazole and voriconazole, depending on the severity of infection $(2,4)$. Daudia and Jones in 2008 reviewed the advances in the management of aspergillosis of paranasal sinuses and concluded that surgery remains the treatment of choice, but itraconazole alone appears to be curative; in fulminant aspergillosis, radical surgery and amphotericin $\mathrm{B}$ continue to be the treatment of choice $(2,5)$.

Endoscopic surgery has been found to be feasible and efficient, enabling excellent local control and less morbidity than traditional approaches (16, 2629). Sinus surgery must be performed as soon as feasible.

However, aspergillosis sometimes extends into the cranial cavity and lungs from paranasal sinuses and in such cases, prognosis is usually very poor $(13,14)$.

The prognosis is directly related to the severity of the underlying disease, the extent of the disease when treatment begins, and the aggressiveness of the treatment.

Both of our cases showed good prognosis and the clinical outcome was satisfactory on follow-up. Repeat fungal culture performed 15 days after treatment was negative for growth of any fungal pathogens.

\section{Conclusion}

Aspergillosis of the paranasal sinuses is usually seen in individuals who are otherwise healthy, so clinicians should be aware that chronic sinusitis that is unresponsive to usual management and which demonstrates a radiodense focus within the antrum is highly suggestive of aspergillosis of the paranasal sinuses. Because the numbers of reported cases are increasing, its inclusion as part of the differential diagnosis in antral disease is important, particularly because not all forms follow a benign course and the prognosis is dependent on early diagnosis. Tissue invasion by the hyphae of $A$. fumigatus must be seen microscopically to establish the diagnosis, but culture is required to identify the fungal species involved. We thus emphasize that early diagnosis 
and prompt treatment are essential to avoid the high morbidity and mortality associated with this destructive disease.

\section{References}

1. Lowe J, Bradley J : Cerebral and orbital aspergillosis infection due to invasive aspergillosis of ethmoid sinus. J Clin Pathol, 39: 774-778, 1986.

2. Washburn RG, Kennedy DW, Begley MG, Bennett JE : Chronic fungal sinusitis in apparently normal hosts. Medicine, 67 : 231-247, 1988.

3. Abdulrahman AA, Mushira E, Zeyad M, Kawther A : Chronic invasive aspergillosis of paranasal sinuses in immunocompetent hosts from Saudi Arabia. Am J Trop Med Hyg, 65 : 83-86, 2001.

4. Young RC, Bennet JE, Vogel CL: Aspergillosis: The spectrum of the disease in 98 patients (Baltimore). Medicine, 49: 147-173, 1970.

5. Fraser RS : Pulmonary aspergillosis: Pathologic and pathogenic features. Pathol Annu, 28: 231-277, 1993.

6. Katzenstein ALA, Sale SR, Greenberger PA: Pathologic findings in allergic sinusitis: A newly recognized form of sinusitis. Am J Surg Pathol, 7 : 439-443, 1983.

7. Myoken Y, Sugata T, Tai Chi K, Fujihara M : Pathologic features of invasive oral, 56: 263-270, 1996.

8. Dreizen S, Bodey GP, McCredie KB, Keating MJ : Orofacial aspergillosis in acute leukemia. Oral Surg, Oral Med, Oral Pathol, 59: 499-504, 1985.

9. Katzenstein A, Sale S, Greenberger P : Allergic aspergillosis sinusitis : a newly recognized form of sinusitis. J Allergy Clin Immunol, 72 : 89-93, 1983.

10. Bozkurt MK, Ozcelik T, Saydam L, Kutluay L: A case of isolated aspergillosis of the maxillary sinus. Kulak Burun Bogaz Ihtis Derg, 18: 53-55, 2008.

11. Garcia MF, Pinilla JC, Echeverria CL, Ferrero AE, Hernandez AV: Invasive maxillary aspergillosis: Report of a case and review of the literature. Medicina Oral, $7:$ 200-205, 2000.

12. Myoken Y, Sugata T, Fujita Y, Fujihara M, Iwato K, Murayana SY, Mikani Y: Early diagnosis and successful management of atypical invasive aspergillus sinusitis in a hematopoitic cell transplant patient: A case report. J Oral Maxillofac Surg, 64 : 860-863, 2006.

13. Kadziora K, Stominski JM, Gil K, Porzezinska M, Gorezewska A: Invasive aspergillosis of paranasal sinuses, lung. and brain. Pol Pneumonol Allergol, 76 : 400-406, 2008.

14. Notani K, Sato C, Hashimoto I, Makino S, Kitada H, Fuckuda $\mathrm{H}$ : Intracranial aspergillus infection from the paranasal sinus. Oral Surg, Oral Med, Oral Pathol Oral Radiol Endod, 89 : 9-11, 2009.
15. Aneesh MK, Seith A, Sharma S: Symptomatic perineural extension of fungal sinusitis in : Imaging Features. Kandpal H, Singapore Med J, 49: e171-174, 2008.

16. Mylona S, Tzavara V, Ntai S, Pomoni M, Thanos L: Chronic invasive sinus aspergillus in an immunocompetent patient: A case report. Dentomaxillofacial Radiol, 36 : 102-104, 2007.

17. Kim DG, Hong HC, Chi JG, Han MH, Choi KS, Han $\mathrm{DH}$ : Cerebral aspergillosis in an immunologically competent patient. Surg Neurol, 40 : 26-31, 1993.

18. Smolansky SJ : Aspergillosis of the paranasal sinuses. Ear Nose Throat J, 57: 320-323, 1978.

19. McGuirt WF, Harrill JA : Paranasal sinus aspergillosis. Laryngoscope, 89 : 1563-1568, 1979.

20. Verweij PE, Kerremans JJ, Voss A, Meis JF : Fungal contamination of tobacco and marijuana. J Am Med Assoc, 284 : 28-75, 2000.

21. Shams AG, Motamedi MHK : Aspergilloma of maxillary sinus complicating an oro-antral fistula. Oral Surg, Oral Med, Oral Pathol Oral Radiol Endod, 96 : 3 $-5,2003$.

22. Castlenuovo P, Gera R, Di Giulio G, Canevari FR, Benazzo M, Emanuelli E: Paranasal sinus mycoses. Acta Otorhinonolaryngol Italica, $20: 6-15,2000$.

23. Matjaz R, Jernef P, Mirela KR: Sinus maxillaries mycetoma of odontogenic origin case report. Braz Dent J, 15 : 248-250, 2004.

24. Stammberger H, Jakse R, Beaufort F : Aspergillosis of the paranasal sinuses: $\mathrm{x}$-ray diagnosis, histopathology, and clinical aspects. Ann Otol Rhinol Laryngol, 93 : 251-256, 1984.

25. Daudia A, Jones NS: Advances in management of paranasal sinuses aspergillosis. J Laryngol Otol, 122 : 331-335, 2008.

26. Eliashar R, Resnick IB, Goldfarb A, Wohlgelernter J, Gross M : Endoscopic surgery for sinonasal invasive aspergillosis in bone marrow transplantation patients. Laryngoscope, 117 : 1-4, 2007.

27. Costa F, Polini F, Zerman N, Robiony M, Toro C, Politi M : Surgical treatment of aspergillus mycetomas of maxillary: Review of literature. Oral Surg, Oral Med, Oral Pathol Oral Radiol Endod, 103 : e23-29, 2007.

28. Chobillon MA, Jankowski R: What are the advantages of the endoscopic canine fossa approach in treating maxillary sinus aspergillomas? Rhinology, $42: 230$ $-235,2004$.

29. Snyderman CH, Pant H, Carrau RL, Pervedello D, Gardner P, Kassam AB : What are the limits of endoscopic sinus surgery?: The expanded endonasal approach to skull base. Keio J Med, 58 : 152-160, 2009. 